\title{
Die Mittelschule im Reformdruck - wo steht die Geographie?
}

Die Mittelschulen sind gefordert. Immer widersprüchlicher werden die gesellschaftlichen Ansprüche an die Schule. Sollen Gymnasien vor allem auf das Hochschulstudium oder letztlich auf das spätere Leben vorbereiten? Allgemeinbildung oder Spezialisierung - das ist die Frage. Oder gar beides? Doch reicht die Zeit dafür? Also: «Mut zur Lücke», «Verwesentlichung des Unterrichts»! Die verunsicherte Institution Gymnasium sucht nach Lösungen. Das immer häufiger genannte Zaubermittel Schulentwicklung soll weiterhelfen. "Von Grund auf erneuern", lautet die Devise.

Es fällt auf, daß in letzter Zeit in den verschiedensten Bereichen des Bildungswesens unter Reformdruck Leitbilder entworfen und neue Konzepte ausgearbeitet werden. Die Notwendigkeit, sich den neuen gesellschaftlichen Herausforderungen zu stellen und angemessen zu reagieren, ist erkannt. In der Bildungslandschaft Schweiz ist derzeit so viel in Fluß wie wohl noch nie! Können wir uns aber diesen großen Aufwand für Planung und Umsetzung von Reformprojekten auf allen Ebenen und an vielen Orten überhaupt leisten? Könnten allenfalls Synergien durch institutionsübergreifende Gesamtkonzepte genutzt werden? Haben die Reformen tatsächlich ihre Wirkung bis in die einzelnen Unterrichtsstunden, von denen ja dann die Schüler in doppelter Hinsicht «leben»: einerseits verbringen sie doch einen beachtlichen Teil ihres Zeitbudgets in der Schule, und andererseits sollten im Unterricht Orientierungshilfen und Qualifikationen zur Bewältigung von "Lebenssituationen» gefördert werden.

Auch der Fachbereich Geographie an Mittelschulen ist gefordert. Reformen machen an den Disziplingrenzen nicht halt, Lehrpläne und Stoffprogramme der Geographie werden evaluiert. Sind beispielsweise topographisches Grundwissen als hälftiger Bestandteil der Maturitätsprüfung oder die Behandlung der Geologie im letzten Jahr vor der Matura noch mit den neuen Bildungszielen kohärent, oder widerspiegeln solche Sachverhalte zementierte schulinterne Traditionen nach dem bekannten Motto "Geography is what geographers do»? Glaubenskriege zwischen Verfechtern physischgeographischer sowie wirtschafts- und sozialgeographischer Ausrichtung sind nutzlos, ja mehr noch: sie erhöhen den ohnehin vorhandenen Legitimationsdruck des Faches Geographie in einer bildungspolitisch sensiblen Phase. Es stellt sich die ernsthafte (Überlebens-)Frage: Wo positioniert sich die Geographie mit welchen Zielen im Kanon der gymnasialen Unterrichtsfächer?

\section{Wie reagiert(e) die (Mittelschul-)Bildungspolitik auf gesellschaftliche Entwicklungen?}

Die Beschleunigung des gesellschaftlichen und wirtschaftlichen Wandels und die damit verbundenen einschneidenden Veränderungen wie beispielsweise neue Informationstechnologien, Verlust an gesellschaftlichem Zusammenhalt und Wertepluralismus führten zu einem akuten Handlungsbedarf. Was gestern noch als festes Grundwissen galt, ist heute schon überholt.

Vor gut 10 Jahren veröffentlichte die Kommission Gymnasium-Universität (KGU, 1985) die "10 Thesen zum heutigen Zweckartikel der Maturitätsanerkennungsverordnung (MAV)». Auszüge aus diesem Dokument (Abb. 1) zeigen, wie - zumindest gedanklich - schon früh auf die veränderten Ansprüche reagiert wurde und diese Thesen deshalb noch heute modern wirken. Diese Thesen stellen zweifellos einen Meilenstein in der schweizerischen Reformgeschichte des Gymnasiums dar und haben die Diskussionen über Entwicklung von Leitbildern und Lehrplänen nachhaltig beeinflußt.

Die Stellungnahme der Philosophisch-Naturwissenschaftlichen Fakultät der Universität Basel zu diesen Thesen weist in die gleiche Richtung, formuliert aber oft noch präziser die zentralen Leitvorstellungen eines modernen Gymnasiums (Abb. 2).

Diese wichtigen Impulse führten in den folgenden Jahren zu einer intensiven Zusammenarbeit von Gymnasiallehrerinnen und -lehrern mit dem Ziel, einen «Rahmenlehrplan für die Maturitätsschulen» zu schaffen. Dank der Weiterbildungszentrale Luzern und dem Ausschuß Gymnasium, die als Stabsstellen der Erziehungsdirektorenkonferenz (EDK) wirkten, konnte dieses gesamtschweizerische Werk 1994 erfolgreich abgeschlossen werden. Die Abb. 3 zeigt im vollen Wortlaut den Rahmenlehrplan für Geographie.

Der Rahmenlehrplan, zunächst nur als Empfehlung verabschiedet, ist nun ins neue eidgenössische Maturitätsreglement (MAR 15.2.95) integriert worden und erhält damit im Sinne eines Planungsinstrumentes verbindlichen Charakter (MAR, Art. 8).

Hans R. Volkart, Dr., Witellikerstraße 39, CH-8702 Zollikon 
(...)

5. Da Freude am Schönen eine fundamentale Komponente von Bildung darstellt, soll den ästhetischen Aspekten in allen Fächern - nicht nur in den sogenannten Kunstfächern - die nötige Aufmerksamkeit geschenkt werden.

(...)

7. Neugierde, Eigeninitiative und Leistungsbereitschaft des Einzelnen sind im Rahmen einer ethisch fundierten Erziehung zu Solidarität und Zusammenarbeit zu entwickeln. Das Verantwortungsgefühl soll, vom inneren Leben der Schule ausgehend, das eigene Land, seine Kultur und seine demokratischen Einrichtungen, aber auch die Zukunft der ganzen Menschheit umfassen. Dazu gehört die Einsicht, daß nicht mehr alles gemacht werden darf, was machbar ist, und daß der Mensch Rücksicht auf Mitmensch und Umwelt zu nehmen hat.

(...)

9. Bildungsvorgänge haben Prozeßcharakter. Bildung muß daher als dynamischer Vorgang verstanden werden, an dem nicht nur das Ziel, sondern die ihn begleitenden Impulse von großer Bedeutung sind. Mancher Anstoß gelangt erst später zur vollen Wirkung. Selbst als grundlegend betrachtete Kenntnisse und Fertigkeiten können sich wandeln; daher ist es wichtig, daß die Schüler in der Entwicklung der Wissenschaften Einblicke gewinnen und so lernen können, auch in Zukunft auf neuartige Herausforderungen beweglich und kreativ zu reagieren.

(...)

Abb.1 Auszüge aus dem Thesenpapier der KGU (1985).

(...)

1. Der gymnasiale Unterricht muß in jedem Fach so allgemein bildend sein, daß er denjenigen Schülern dient, die in ihrer späteren Ausbildung diesem Fach nicht mehr begegnen und aus ihrer Erfahrung im Gymnasialunterricht ihre Kenntnisse und ihr bleibendes Bild von diesem Wissensgebiet erhalten.

2. Wichtiger als Vollständigkeit des Wissens gemäß einem vorgegebenen Stoffplan (die doch nie erreicht werden kann) und der Kenntnis einer Fülle von Einzelfakten ist Verständnis für die für das jeweilige Fach charakteristische Art des Denkens und Problemlösens, demonstriert anhand ausgewählter typischer Beispiele, die dafür um so sorgfältiger erarbeitet werden soll.

(...)

4. Der Gymnasialunterricht soll auch beim zukünftigen Studenten des Fachgebiets in erster Linie das Interesse für das Fach und die Freude wecken, den in diesem Gebiet sich zeigenden Fragen nachzugehen, Probleme zu lösen - und zwar selber zu lösen zu versuchen und nicht Fragestellung wie auch ihre Lösung vorgesetzt zu erhalten.

$(\ldots)$

Abb.2 Auszüge aus der Stellungnahme der Philosophisch-Naturwissenschaftlichen Fakultät der Universität Basel (1988).

\section{A. Allgemeine Bildungsziele}

Durch den Geographieunterricht gelangen die Schülerinnen und Schüler zur Einsicht, daß Lebensansprüche, Normen und Haltungen raumprägend sind. Dies soll zu einem verantwortungsbewußten Umgang mit dem Lebensraum führen.

Schülerinnen und Schüler lernen eine Landschaft in ihrer Ganzheit bewußt erleben und sie mit Hilfe geographischer Methoden und Kenntnisse analysieren. Sie sind fähig, sich auf der Erde mit ihren vielfältigen Strukturen zu orientieren. Sie begegnen der Welt, insbesondere anderen Kulturen, mit Offenheit.

Das Zusammenwirken und die gegenseitige Beeinflussung von Mensch und Natur soll verständlich werden. Veränderungen der Lebensräume sind zu erfassen und zu beurteilen.

Die Geographie enthält Elemente natur- und humanwissenschaftlichen Denkens; deshalb verbindet sie die beiden Bereiche. Sie fördert das Erkennen von Zusammenhängen und regt die fächerübergreifende Behandlung von Themen an. 


\section{B. Begründungen und Erläuterungen}

Der verantwortungsbewußte Umgang mit dem Lebensraum ist das bedeutendste Bildungsziel des Geographieunterrichtes. Verantwortungtragen setzt voraus, daß die Schülerinnen und Schüler komplexe Landschaftsgefüge verstehen. Früher mußte sich der Mensch weitgehend an seine Umwelt anpassen. Heute prägt er die Umgebung in hohem Maße, kann aber die Konsequenzen seines Wirkens oft nur mangelhaft erfassen. Eine Auseinandersetzung mit den durch den Menschen verursachten Veränderungen des Lebensraumes ist deshalb notwendig. Geographie wird wesentlich umfassender verstanden, als in der ursprünglichen Wortbedeutung (Geographie = Erdbeschreibung) zu erkennen ist. Je nach Betrachtungsweisen stehen abgegrenzte Landschaftsräume, wie Regionen oder Kontinente, oder thematische Frage- und Problemstellungen zur Diskussion.

Die Ganzheit eines Raumes setzt sich aus einzelnen Teilen (sogenannten Geofaktoren) zusammen, die durch intensive Wechselbeziehungen miteinander verknüptt sind. So sind Grundkenntnisse, z. B. in Geologie, Geomorphologie und Klimatologie, wichtig, um die Naturgrundlagen einer Landschaft erkennen und beurteilen zu können. Mit der Bedeutung des Menschen als Gestalter einer Landschaft wird dessen Wirken zu einem zentralen Untersuchungsobjekt. Kulturgeographie-Grundkenntnisse, z. B. aus den Bereichen Ökonomie und Ökologie, sind deshalb notwendig. Exkursionen sind ein wichtiger Bestandteil des Unterrichtes, um diese Inhalte erfahrbar und anschaulich zu machen.

Um sich in den vielfältigen Strukturen (Verteilungen, Zusammenhänge, Ordnungen) orientieren zu können, braucht es vernetztes Denken. Die Geographie liefert einen wichtigen Beitrag zur Interdisziplinarität, weil Querverbindungen zu verschiedenen Fachdisziplinen der Natur- und Geisteswissenschaften bestehen.

In der Geographie werden den Schülerinnen und Schülern wesentliche Kenntnisse, Fertigkeiten, Einsichten und Haltungen vermittelt, die ihnen helfen, ihre Rechte und Pflichten als Staatsbürger und Staatsbürgerinnen wahrzunehmen und verantwortungsbewußt zu handeln.

\section{Richtziele}

\section{Grundkenntnisse}

- Die fachspezifischen Grundbegriffe kennen.

- Über ein topographisches Grundwissen verfügen, um aktuelle Ereignisse geographisch deuten und einordnen zu können.

- Landschaftselemente und ihre raumprägenden Faktoren kennen.

- Die Prozesse des Landschaftswandels erfassen.

- In Grundzügen die Geologie der Schweiz kennen.

\section{Grundfertigkeiten}

- Karten lesen und sich im Gelände zurechtfinden.

- Geographische Darstellungsmethoden anwenden, thematische Karten, Profile, Diagramme, Statistiken, Modelle, Bilder und Texte interpretieren und z. T. selbst entwerfen; Ergebnisse geographischer Untersuchungen verständlich darstellen und weitergeben.

- In Modellen und Fallbeispielen geographische Faktoren erkennen und Prozesse verstehen.

- Landschaftselemente, ihre Wechselwirkungen und Strukturen beobachten, erkennen, interpretieren und beurteilen:

- Ursachen und Zusammenwirken von Naturkräften erkennen.

- Beziehungen zwischen natur- und kulturgeographischen Elementen erfassen.

- Die Wechselwirkungen zwischen den Daseinsfunktionen des Menschen (Wohnen, Arbeiten, Freizeit) und Umwelt verstehen und beurteilen.

- Erkennen, wie Standortfaktoren die wirtschaftliche Nutzung einer Landschaft bestimmen.

- Bedeutung der gesetzlichen Vorschriften und Folgen ihrer Veränderung für die Landschaft abschätzen.

- Kulturelle und gesellschaftliche Einflüsse auf Raumnutzung und Raumentwicklung erkennen.

- Die zunehmende Verflechtung von Ländern und Kulturen und die daraus resultierenden Veränderungen der Lebensbedingungen einsehen.

\section{Grundhaltungen}

- Die Begegnung mit anderen Menschen, Kulturen und Landschaften als Bereicherung erfahren und durch Vergleiche die eigene Umwelt besser verstehen.

- Durch persönliche Erlebnisse und Erfahrungen seine Einstellung überdenken, sich auftauchender Probleme bewußt werden und sich für deren Lösung einsetzen.

- Bereit sein, persönliche raumwirksame Tätigkeiten zu hinterfragen, und entsprechend verantwortungsbewußt handeln. 


\section{Wie reagiert(e) die (Mittelschul-)Geographie auf diese Bildungsinitiativen?}

Betrachtet man einerseits die Forderung nach umfassender Bildung und andererseits den Inhalt des Rahmenlehrplanes in Geographie, läßt sich leicht herauslesen, daß ein Großteil der Aufgaben zunächst nicht so sehr im fachdidaktischen, sondern vor allem im allgemeindidaktischen Bereich zu lösen sind. Auch HENNINGS (1993, S. 130) konstatiert, es gehe künftig (auch im Geographieunterricht) «um

- Begrenzung des spezialisierten Fachwissens,

- Ausbau einer ganzheitlichen Allgemeinbildung mit fächerübergreifender Struktur,

- Förderung von Selbständigkeit, Verantwortlichkeit, Teamgeist und Kooperation, Kommunikationsfähigkeit, Kreativität und Flexibilität,

- Schaffung eines Theorie- und Methodenbewußtseins, das die Einspurigkeit des kausal-linearlogischen Denkens in Richtung eines komplexere Strukturen erfassenden vernetzten Denkens ausweitet,

- systematische Einbeziehung einer Produkt- und Handlungsorientierung, die wissenschaftliches Denken an Problemlösungsstrategien heranführt,

- Förderung von sozialer Handlungskompetenz und politischem Engagement, indem Wissen in seinen historischen Entstehungsbedingungen nachvollziehbar und in seiner praktischen Anwendung im Hinblick auf soziale und ökologische Folgewirkungen kritisch überprüfbar gemacht wird».

Allerdings - und dies ist die harte «Knochenarbeit» für jeden einzelnen (Geographie-)Lehrer - braucht die konkrete Umsetzung dieser Anliegen für den Schulalltag viel Kraft und Ausdauer. Ein erneut aufkommender rauher Wind der Legitimation bläst entgegen. Es geht um Stundendotationen. Wie viele Stunden bleiben dem Fach Geographie? Denn es ist zu bemerken, daß Geographie zusammen mit Geschichte sowie dem neuen obligatorischen Fach Wirtschaft und Recht dem neu geschaffenen Bereich Geistes- und Sozialwissenschaften zugeordnet wurde. Diese drei Fächer sollen zusammen zwischen 10 und 20\% Zeitanteil im Grundlagenunterricht erhalten. Wer hat da das bessere Lobbying? Bei der Zuteilung der Anteile dürfte auch das Image eines Faches eine erhebliche Rolle spielen. Erfahrungen mit dem Geographieunterricht in der eigenen Schulzeit beeinflussen bekanntlich wesentlich eine potentielle Unterstützung von seiten der Verwaltung, der Aufsichtsbehörden und der Öffentlichkeit.

Die negative Einstellung und Kritik gegenüber einer frontalen, dozierenden Wissensvermittlung ist aufgrund eigener Schulerfahrung verbreitet. Weil diese Unterrichtsform heute aber vielerorts noch gepflegt wird, ist diese Kritik berechtigt. Denn wer Wissen als «Faktenwissen» oder "Bücherwissen» versteht, entwertet Unterrichtsinhalte zur bloßen Kenntnis, d. h., der Prozeß der Wissenaneignung entartet in ein Auswendiglernen. Was soll in einer Geographieklausur das Überprüfen von bei- spielsweise 50 verschiedenen morphologischen Begriffen, wenn der Schüler damit wohl seine von der Wandtafel übernommenen Hefteinträge allenfalls korrekt wiedergeben kann, aber seine Leistung ohne jeden intellektuellen, kreativen, sinnlichen oder praktischen Bezug erbringt. Schon 1915 sagte DEWEY (S. 249, zit. in LAND. WEHR 1994, S. 12): «An Anhäufung und Aneignung von Wissen zum Zwecke der Wiedergabe bei Wiederholungen und Prüfungen wird zuviel getan. Wissen im Sinne von wirklicher (Erkenntnis) bedeutet das arbeitende Kapital, das unentbehrliche Hilfsmittel für weiteres Forschen, für das Erkennen und Erlernen neuer Stoffe und Dinge. Häufig wird es jedoch als Selbstzweck behandelt, und die Aufgabe der Schüler besteht darin, es aufzuhäufen und auf Anfrage auszubreiten. Dieses statische «Speicher-Ideal des Wissens ist der Feind echter erzieherischer Entwicklung. Es bewirkt nicht nur, daß Gelegenheiten zum Denken ungenützt vorübergehen, sondern schwemmt geradezu das Denken hinweg."

Mit Recht weist LANDWEHR in seinem kürzlich erschienenen Buch daraufhin, daß von der "Kenntnisvermittlung" endlich zur «Erkenntnisvermittlung» übergegangen werden sollte: "An Stelle einer rquantitativen (kenntnisorientierten) Wissensvermittlung) muß eine (qualitative (erkenntnisorientierte) Wissensvermittlung) treten, die das Individuum zum schöpferischen Umgang mit dem erworbenen Wissen befähigt und so die selbständige Transformation des erworbenen Wissens auf neue bzw. veränderte Zusammenhänge ermöglicht.» $\mathrm{Um}$ am oben erwähnten Beispiel anzuknüpfen: Statt 50 geomorphologische Begriffe als «leere Hülse» zu lernen und auswendig wiederzugeben, müßte an einem konkreten, auch möglichst anschaulichen, «erfahrbaren» Raum z. B. der Formenschatz einer Flußlandschaft erarbeitet werden (Mäander im Sohlental mit Gleit- und Prallhang, Schotterterrassen usw.). Anschließend kämen der Transfer und eine allfällige Modifikation der Regeln und Gesetze aufgrund veränderter Bedingungen. Der Paradigmenwechsel könnte einfach ausgedrückt auch mit «mehr Tiefe statt Breite» umschrieben werden.

\section{Welche Konsequenzen sind für den zukünftigen Geographieunterricht an Gymnasien zu ziehen?}

In den Augen des Schreibenden dürften folgende vier Komponenten eine angepaßte und nachhaltige Entwicklung im geographischen Schulalltag bestimmen und damit Gegenstand einer "neuen Lernkultur» sein.

Erstens gilt es Abschied zu nehmen vom systematischen Lernen. Gleiche Titelnumerierungen (3.2.5 Die glaziale Erosion) im Schülerheft wie in den Vorlesungsunterlagen der Universität sind suspekt. Ausgedünnte, d. h. didaktisch reduzierte Inhalte der Vorlesungsskripten müssen verschwinden. Gesucht sind exemplarisch ausgewählte Themenbereiche (z. B. Umweltbelastungen 
im Alpenraum), in denen detailliert und sorgfältig gearbeitet wird. Dabei spielen genaues Beobachten und Wahrnehmen, eigenes Entdecken und Erforschen eine zentrale Rolle. Es sind also zunächst Fragen zu formulieren und Lösungen zu suchen, bevor der Lehrer mit seinem Wissen eingreift (zuerst die Praxis, dann die Theorie).

Die schwerpunktmäßig ausgewählten Stoffgebiete sollen - zweitens - Aktualitätsbezug besitzen, Schülererfahrungen einbeziehen und das Einüben von Grundfertigkeiten ermöglichen. Von großer Bedeutung sind heute sicher Umwelt- und Disparitätenfragen. Die Arbeitsgruppe Fachdidaktik (AFGg), ein Gremium, das alle an schweizerischen Hochschulen Geographiedidaktik lehrenden Dozentinnen und Dozenten umfaßt, ist zurzeit damit beschäftigt, in Übereinstimmung mit dem Rahmenlehrplan und den neusten didaktischen Erkenntnissen einen Modell-Lehrplan zu verfassen. Dabei zeigt sich übrigens, daß mit einem gemischt thematisch-regionalen Ansatz das Verhältnis zwischen physischgeographischen und wirtschafts- und sozialgeographischen Inhalten etwa eins zu zwei betragen dürfte. Die Fachdidaktiker sind überzeugt, mit diesem Arbeitsinstrument eine Hilfe zur Entrümpelung der z.T. sehr veralteten schulinternen Lehrpläne anzubieten, und hoffen auf rege Benützung im Interesse unseres Faches und in Verantwortung gegenüber unseren Schülern.

Drittens lassen sich diese gut evaluierten Inhalte aber, sollen sie u. a. den Prinzipien vom entdeckenden Lernen, von Selbsttätigkeit und Teamarbeit genügen, nur mit Unterrichtsformen realisieren, die sich vom herkömmlichen Frontalunterricht mit Unterrichtsfragen und eingestreuter Gruppenarbeit wesentlich unterscheiden. $\mathrm{Zu}$ den unter dem Begriff «erweiterte Lernformen" (ELF) zusammengefaßten Methoden gehören z. B. Werkstattunterricht, Arbeit mit Leitprogrammen, Lernen an Projekten und Fallstudien. Ziel dieser Unterrichtsmethoden ist es, nicht nur einen kognitiv und instrumentell anspruchsvolleren und damit fordernden Unterricht zu gestalten, sondern auch Lernwege zu variieren. Kein Mensch lernt gleich wie der andere. In jeder Klasse sitzen verschiedene Lerntypen. So besteht die Möglichkeit, einzelnen jungen Menschen gerechter zu werden. Es ist fast müßig zu sagen, daß sich die Geographie für die Umsetzung dieser Ideen und Formen ganz besonders eignet!

Allerdings ist - viertens - diese Umsetzung an Bedingungen geknüpft. Eine Bedingung ist, Abschied zu nehmen vom traditionellen "45-Minuten-Takt-Unterricht», der die Schüler täglich mit 7-9 verschiedenen Stoffgebieten konfrontiert. An seine Stelle muß eine Unterrichtsorganisation treten, die bei Fächern mit kleinen Pensen wie z. B. Geographie (zwei Jahresstunden) konsequent auf Doppelstunden setzt und Jahrespensen auf Semester bzw. Semesterpensen auf Quartale konzentriert. Erst die Gestaltung von zwei Doppelstunden pro Woche und Klasse gibt die Möglichkeit, mit den ELF zu arbeiten. Zusätzlich sind häufiger Studienwochen zu organisieren, in denen "vor Ort», d. h. im Feld oder Labor, praktisch ent- deckt und geforscht werden kann. Der Schreibende unterrichtet an einer Schule, in der pro Jahr und Schulklasse der Sekundarstufe II vier Arbeitswochen stattfinden. Erfahrungen zeigen, daß nicht nur die Schüler motivierter mitarbeiten, sondern daß sie gleichzeitig durch die inhaltliche Konzentration auch intellektuell ansprechendere Leistungen erbringen. Wenn sich gar noch Kolleginnen und Kollegen zum interdisziplinären Teamteaching zusammenfinden, ist dies ein großer Schritt in die richtige Richtung. Klassenübergreifend mit Maturaklassen während vier Monaten in den Fächern Geschichte, Religion, Englisch und Geographie an einem Thema (z. B. «Indien») zu arbeiten, wie es der Schreibende erleben konnte, macht das Unterrichten wesentlich attraktiver. Eine zweite Bedingung ist in der Qualität der Lehrerarbeit zu suchen. Wie Untersuchungen in der Wirksamkeitsforschung von Unterricht belegen, sind klare Zielsetzungen, hohe Leistungserwartungen und starkes schulbezogenes Engagement wichtige Prämissen «guten» Unterrichts. Auch die renommierte «Neue Zürcher Zeitung» betitelte zu Recht ihre redaktionellen Kommentare zur laufenden Maturitätsreform mit «Die Weichen stellt der Lehrer» oder «Schulqualität entsteht in den Köpfen der Lehrer» (1995). Dem ist beizufügen, daß letztlich Reformprojekte von den Haltungen der Lehrkräfte abhängen. Ein Lehrer, der schwer zu bewegen ist, an einem seiner schulfreien Tage einen Weiterbildungskurs zu besuchen, hat die falsche Einstellung, und der bittere Satz in einem der oben erwähnten «NZZ»-Kommentare, wonach «Lehrer nur beschränkt lernfähig» seien, bekäme seine Bestätigung.

An diesem Punkt kann eine dritte Bedingung angeknüpft werden: Heutige Gymnasien brauchen in der Schulleitung starke Führungskräfte, Rektoren also, die reformfreudige Lehrerinnen und Lehrer unterstützen und als Personalchefs letztlich die Verantwortung für den Unterricht an ihrer Schule tragen (Schulbesuche durchführen; Verbesserungsmaßnahmen vorschlagen und begleiten) und zudem in offenem, auch institutionalisiertem Kontakt zur Schülerschaft stehen. Damit kann sich ein animierendes Klima für alle Beteiligten entwickeln und Schulkultur («corporate identity») wachsen, von der noch zu oft nur geträumt wird.

\section{Ein Ausblick}

Diskussionen, ob Geographie als "Zentrierungsfach» oder als "Schlüsselfach» angesprochen werden kann oder nicht, sind unerheblich im Vergleich zur Bedeutung einer nachhaltigen Förderung geographischer Bildung an der Basis, d.h. im Geographieunterricht und durch Erfahrungen im Alltag. Der geographische Raum ist Voraussetzung und Bestimmungsgröße unseres Lebens. In ihm vollziehen sich alle bedeutsamen Tätigkeiten des Menschen. Die Veränderungsprozesse in Gesellschaft und Wirtschaft bedingen veränderte Ansprüche an geo- 
graphische Räume und verschärfen Nutzungskonflikte. Mit Recht wird in einem jüngst veröffentlichten Positionspapier des Verbandes Deutscher Schulgeographen gesagt, daß das Fach Geographie «die Schülerinnen und Schüler in die Lage versetzt,

1. ein wissenschaftlich fundiertes Bild von der Erde und den Wechselwirkungen zwischen Mensch und Raum zu entwickeln und

2. sich verantwortungsbewußt an der Bewältigung von Gegenwartsproblemen und Zukunftsaufgaben zu beteiligen» (Verband Deutscher Schulgeographen, 1995).

Die jetzige Umbruchphase in den schweizerischen Gymnasien kann auch als die große Chance für das Fach Geographie interpretiert werden. Eine offensivere Selbstdarstellung (Entwurf neuer Lehrpläne, Integration der erweiterten Lernformen, Gestaltung von Schulanlässen), gekoppelt mit der Bereitschaft zur Fortbildung (was auch Zusammenarbeit mit Kolleginnen und Kollegen beinhaltet), macht den Beruf der Geographielehrerin und des Geographielehrers wohl anspruchsvoller, aber letztlich sicher befriedigender.

\section{Verwendete Quellen}

Arbeitsgruppe Fachdidaktik Geographie, Modell-Lehrplan Geographie für die Sekundarstufe II, AFGg-Dokument Nr. 4 / 1995.

Erziehungsdirektorenkonferenz (EDK), Rahmenlehrplan für die Maturitätsschulen, Bern 1994.

HENNINGS, W., Strukturwandel und Bildungsreform, Überlegungen zu einer Revision der Curricula - auch in der Geographie, in: Geographie und ihre Didaktik 3/93.

KGU (Kommission Gymnasium-Universität des Vereins Schweizerischer Gymnasiallehrer), 10 Thesen zum heutigen Zweckartikel der Maturitätsverordnung. In: Gymnasium Helveticum $2 / 85$.

LANDWEHR, N., Neue Wege der Wissensvermittlung, Aarau 1994.

"NZZ", Die Weichen stellt der Lehrer, 18.1.1995.

Philosophisch-Naturwissenschaftliche Fakultät der Universität Basel, Stellungnahme zu den 10 Thesen der KGU, 14.6.88 (Manuskript).

Verband Deutscher Schulgeographen, Positionspapier zur Stellung der Geographie als Schulfach. In: Praxis Geographie $5 / 95$. 